\title{
BENEFÍCIOS DE UM LABORATÓRIO DE INDÚSTRIA 4.0 NO ENSINO SUPERIOR
}

\author{
Kelvin Ricardo dos Santos Vaz-kelvin.vaz@facens.br \\ Alessandro Bogila - alessandro.bogila@facens.br \\ Antonio Carlos Gomes Junior - antonio.gomes@facens.br \\ Marcelo Mendes de Aguiar-marcelo.aguiar@facens.br \\ DenisBorg-denis.borg@facens.br \\ Centro Universitário Facens, \\ Rodovia Senador José Ermírio de Moraes, 1425 - Alto da Boa Vista \\ 18087-125 - Sorocaba - SP
}

\begin{abstract}
Resumo: O conceito de indústria 4.0 engloba diversas tecnologias, todavia para estas serem desenvolvidas em um novo ecossistema, precisam conter uma estrutura adequada. O conteúdo dessa pesquisa visa demonstrar as vertentes estruturais contidas em uma instituição de ensino brasileira, levando em consideração como esta auxilia na evolução de uma organização tendo em vista se tornar referência no mercado formador. O Centro Universitário Facens contempla um laboratório de indústria 4.0, que será utilizado como base para o desenvolvimento desse estudo de caso, atentando para como as universidades transmitem o conhecimento para criar líderes 4.0. Foram analisados os cursos de graduação e pós-graduação da instituição, no que se refere ao ensino de tecnologias 4.0, 270 alunos usufruíram da infraestrutura nos anos de 2018 e 2019. Os cursos de graduação que desfrutaram do laboratório foram de engenharia mecânica, produção e mecatrônica, já no que se refere a pós-graduação, os cursos de manufatura avançada 4.0 e controle e automação marcaram presença nesse ambiente tecnológico. Houve um crescimento de aproximadamente $55 \%$ dos alunos impactados levando em conta os anos de 2018 e 2019. A metodologia ativa de aprendizagem foi um dos fatores que mais contribuiu para a melhoria do método de ensino dentro do laboratório e da instituição como um todo, o ambiente foi projetado totalmente voltado ao ensino, desde as configurações das mesas até o planejamento das aulas. O espaço também contribuiu para a elaboração de projetos acadêmicos, que foram ao total, 31 projetos propostos, em que 16 foram concluídos e 15 estão em desenvolvimento.
\end{abstract}

Palavras-chave: Indústria 4.0. Ensino. Mercado. 


\section{INTRODUÇÃO}

A indústria 4.0 é um termo frequentemente usado na Europa, mais recorrente na Alemanha. Esse conceito foi seguido das três revoluções industriais anteriores, originando o 4.0 (MÜLLER; BULIGA; VOIGT, 2020). Semelhantemente em outras revoluções que tinham o seu propósito tecnológico, como por exemplo a terceira revolução com a automatização, a indústria 4.0 não fica atrás, trazendo em sua base principal o Cyber-Physical Systems (CPS), modernização que aborda a interligação de componentes físicos e dados para realizar determinada atividade ou processo.

Em suma, a indústria 4.0 é um conceito de optimização de processos utilizando tecnologias interconectadas com o objetivo de aumentar a eficiência, onde hardware funciona como um serviço gerando dados, assim como parâmetros que podem ser analisados para a optimização e agregação de valor.

O Centro Universitário Facens dispõe de um laboratório de manufatura avançada 4.0 (LMA 4.0), que contempla alguns dos chamados "pilares 4.0", quais são definidos por Leal, Fleury e Zancul (2020) como sendo análise de dados, robótica, simulação, integração de sistemas, internet das coisas, cyber security, cloud computing, manufatura aditiva e realidade aumentada. A instituição detém cursos de graduação e pós-graduação em que alguns destes tópicos são explorados. O laboratório analisado nessa pesquisa foi inaugurado em junho de 2018 em parceria com a empresa FESTO, tendo por objetivo principal promover o conhecimento para formar profissionais capacitados nesse novo âmbito profissional.

No decorrer dessa pesquisa serão mostrados os resultados obtidos com o uso do laboratório voltado a indústria 4.0, evidenciando como um ambiente prático auxilia na aprendizagem e no desenvolvimento de projetos.

\section{VISÃO DE MERCADO E INAUGURAÇÃO DO LABORATÓRIO}

A Indústria 4.0 não se limita somente a digitalização. A combinação de diversas tecnologias habilitadoras transformará a maneira como as empresas pensam e geram os seus negócios, como se posicionam na cadeia de valor, como desenvolvem novos produtos e serviços, ações de marketing e outras frentes (COSTA, 2017). As principais inovações que ocorreram na transformação digital exigem que os agentes empresariais tenham um nível de conhecimento de difícil acesso. O conhecimento vem em formas distintas como (tácito, explícito, aprendido individualmente ou através da aprendizagem coletiva) e está em estreita ligação com a gestão virtual das empresas (COSTACHE et al., 2017).

Observando a demanda de mercado a instituição busca instruir profissionais para que eles estejam aptos a esse novo ecossistema 4.0. Nesse âmbito, o centro universitário projetou como oportunidade a implantação um novo laboratório em seu campus em 2018, a organização fechou uma parceria com a empresa FESTO e o instituto FRAUNHOFER com o objetivo de ser a detentora de uma nova vertente de ensino.

Uma das implementações foi a célula de manufatura da Didactic (linha de segmento da empresa FESTO), que é uma fábrica de aprendizagem utilizada para pesquisa ou desenvolvimento de competência, permitindo que os usuários dominem situações complexas e desconhecidas (ABELE et al., 2015). A linha de aprendizagem 4.0 direciona que a transferência de conteúdo aprendido externamente ao local de trabalho geralmente está associada a enormes perdas, que aponta para as ineficiências em relação às abordagens tradicionais de ensino e aprendizagem, visto que os processos industriais cada vez mais se tornam dinâmicos e 
complexos, nos quais as teorias e métodos de ensinos ultrapassados de tornam ineficazes para essa nova era industrial (SCHUSTER et al., 2015). Por esse motivo, a instituição também pretende desenvolver um centro de estudos 4.0, para atender as demandas da indústria quanto à formação acadêmica e soluções de implantação em plantas antigas ou futuras.

\section{APROXIMAÇÃO ENTRE UNIVERSIDADE E EMPRESA}

A indústria 4.0 é uma grande vertente para a aproximação entre a universidade e indústria, o que pode alavancar o desenvolvimento de ambas as partes, porque a primeira possui a pesquisa e o desenvolvimento tecnológico, e a segunda detém da alta escala e a necessidade de desenvolvimento econômico. Todavia, mesmo que a parceria seja benéfica para ambos os lados existe a limitação do tempo. As empresas esperam um retorno econômico muito rápido e a universidade muitas vezes precisa de um tempo maior para buscar o conhecimento e desenvolvê-lo o que corrobora em um conflito (SEGATTO-MENDES, 1996).

O território nacional tem passado por experiências econômicas negativas nos últimos anos. Isso porque a indústria brasileira não possui um desenvolvimento tecnológico nacional próprio e acaba dependendo de importação tecnológica. Segundo dados levantados pelo Industrial Research Institute (IRI) dos Estados Unidos, as empresas de países desenvolvidos estão investindo menos em laboratórios internos e desenvolvimento de pesquisas e estão buscando mais parcerias com universidades (TARALLI, 1995).

Com o objetivo de investigar o "ganha a ganha" de ambos se fez uma pesquisa pontual sobre os benefícios gerados para as partes (SEGATTO-MENDES, 1996).

a) para as universidades:

- realização da função social da universidade;

- obtenção de conhecimentos práticos sobre os problemas existentes;

- incorporação de novas informações aos processos de ensino e pesquisa;

- obtenção de recursos financeiros adicionais;

- obtenção de recursos materiais adicionais;

- prestígio para o pesquisador;

- divulgação da imagem da universidade.

\section{b) para as empresas:}

- acesso aos recursos humanos altamente qualificados da universidade;

- resolução de problemas técnicos que geraram a necessidade da pesquisa;

- redução de custos e riscos envolvidos em projetos de pesquisa e desenvolvimento;

- acesso a novos conhecimentos desenvolvidos no meio acadêmico;

- identificação de alunos para recrutamento futuro.

É possível observar que as empresas têm necessidade de conhecimento sobre tecnologia e buscam recursos humanos qualificados e o laboratório acaba suprindo algumas dessas demandas solicitadas pela indústria. 


\section{MÉTODOS DE APRENDIZAGEM}

A adaptação a uma nova era de ensino como a Indústria 4.0 não é uma tarefa fácil, todas as áreas de aplicação futuramente terão que lidar direta ou indiretamente com essa nova tecnologia.

Um processo de aprendizagem consiste em quatro etapas: A primeira delas é quando depara se com uma nova experiência chamada pelo autor de "concrete experience", a segunda etapa é a reflexão dessa nova experiência implicando no entendimento, a terceira etapa se conclui como uma idealização da reflexão, a partir desta pode se aprender com a experiência adquirida na primeira etapa, a última etapa visa aplicar o aprendizado para a formação de novos conceitos (KOLB, 1984). A Figura 1 mostra o ciclo de aprendizagem mais detalhadamente.

Figura 1 - Ciclo de aprendizagem por Kolb.

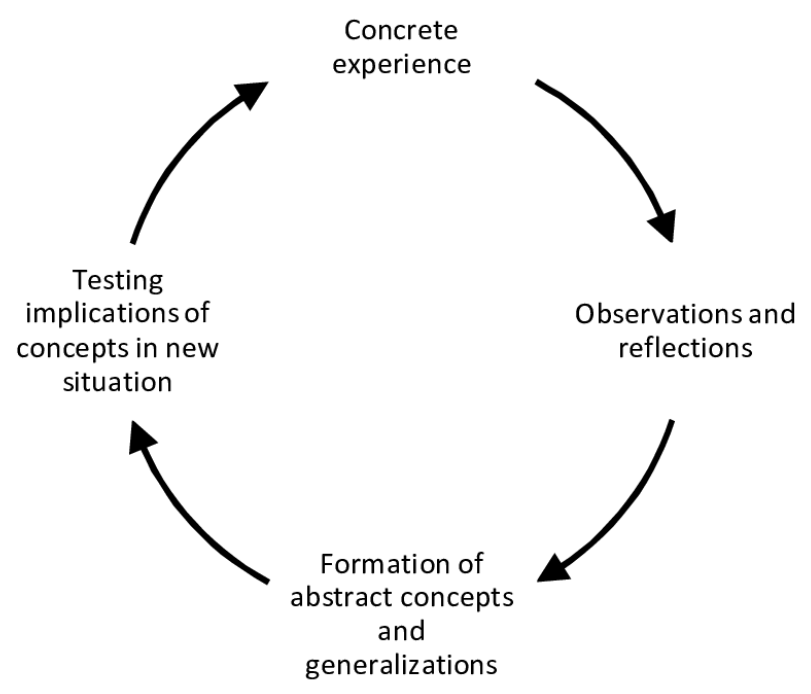

Fonte: Kolb, 1984.

O laboratório se encaixa no processo de aprendizagem em algumas etapas: na primeira delas, o método proporciona experiência concreta para os alunos, contendo a maioria da tecnologia necessária para o aprendizado da indústria 4.0. Consequentemente, junto ao ensino dos professores da instituição que tendem a formar conceitos de aprendizagem, por fim, aplicam a aprendizagem junto a infraestrutura do laboratório.

Essa metodologia tem sido aplicada na organização e as grades curriculares têm sido reformuladas com base na nova demanda de conhecimento que o mercado de trabalho exige.

\section{METODOLOGIA ATIVA E DIFICULDADES DE IMPLANTAÇÃO}

Existe uma grande quantidade de métodos de aprendizagem, todavia no que se refere à metodologia ativa, que como seu próprio objetivo, visa a integração da pesquisa, orientação e trabalho em grupo, no qual os alunos criam o link para a própria aprendizagem.

Devido à dificuldade e erros que podem ocorrer na aplicação dessa metodologia, muitas instituições ficam presas ao modelo antigo de ensino, onde a defasagem de aprendizado é 
grande. Uma provável causa para tal dificuldade é que os professores ficam inseguros de seguir um novo modelo de aprendizado, de se adaptar a este, de cometer erros e aprender com eles, esse modelo ainda não é bem-visto pela sociedade acadêmica (PUNDAD, 2008).

No entanto, as universidades de grande influência na comunidade acadêmica cada vez mais utilizam esse modelo de aprendizagem e veem obtendo grandes resultados melhorando a aprendizagem de seus alunos. A metodologia ativa de aprendizagem segue um termo chamado de "construtivismo", que contrapõe à ideia da aprendizagem por memória, que se baseia no modelo antigo de aprendizagem. Em resumo o construtivismo se ramifica em dois modelos, o cognitivo e o social, onde objetivo de ambos se destaca pelo entendimento como processo de aprendizagem e não pela memorização. $O$ construtivismo não pode ser visto como um modelo, mas ele deve ser utilizado como um guia, isso porque as situações de ensino divergem algumas vezes (KOLB, 1984). A Figura 2 mostra as mesas do LMA 4.0 organizadas com capacidade de até seis alunos para as atividades práticas.

Figura 2 - Organização do ambiente do LMA 4.0.

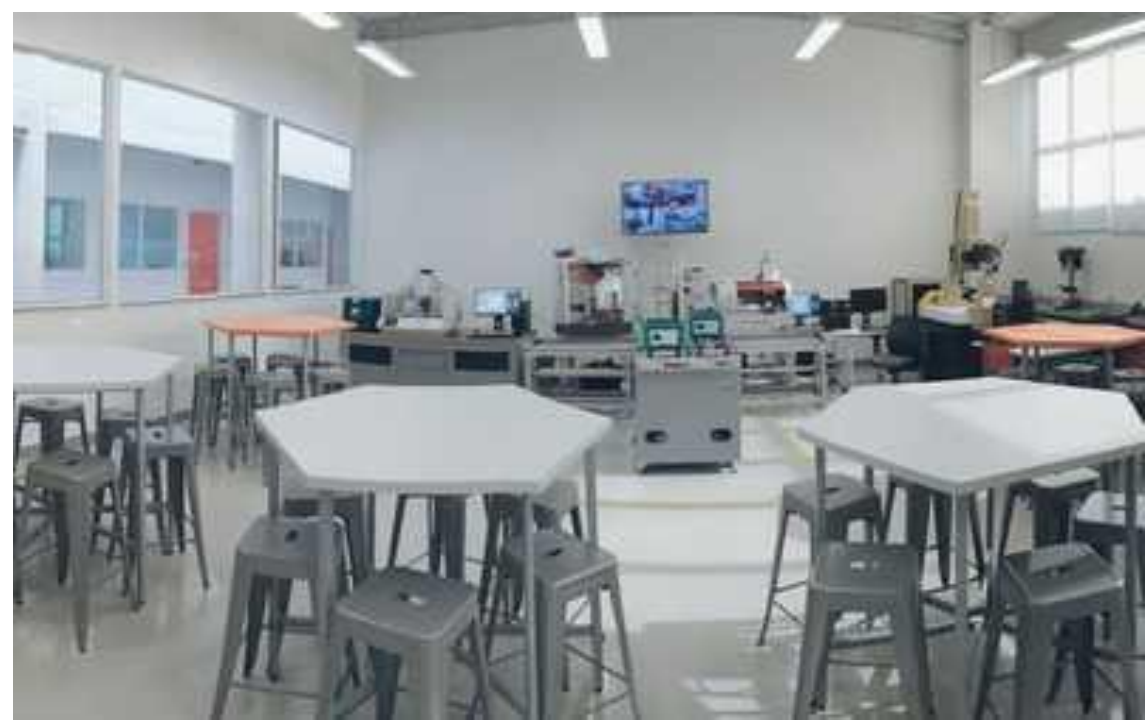

Fonte: Autoria própria.

O modelo do laboratório foi idealizado com o objetivo de aplicar essa metodologia, onde as mesas são organizadas de forma que os alunos possam interagir entre si, tendo a orientação dos facilitadores como base, além de usufruir da estrutura do laboratório durante as aulas.

Além da organização como objetivo da aplicação da metodologia, o laboratório possui 6 notebooks com acesso à internet para os alunos realizarem a pesquisa durante a aula. A metodologia ativa também tem sido aplicada a outros projetos dentro do laboratório como, artigos científicos, Trabalhos de Conclusão de Curso, Semana da Engenharia, entre outras.

Em suma, a aplicação da metodologia ativa de aprendizagem trouxe uma visão positiva dentro da instituição, porque teve um retorno positivo de seus alunos que mostraram um maior aprendizado das aulas no laboratório.

\section{EQUIPAMENTOS E INTERAÇÃO A NOVAS METODOLOGIAS PRÁTICAS}

O laboratório contempla uma célula de manufatura idealizada em parceria com a empresa FESTO, apresenta duas máquinas CNC (torno e fresadora), sistema de docas, um braço robótico e óculos de realidade virtual. A célula também possui um IHM (interface homem-máquina) que 
exibe todas as ações da célula em tempo real, o sensoriamento presente é o ótico que interligam pistões e um sensor RFID (Radio Frequency Identification) que está ligado ao PLC (Programmable Logic Controller) comandado por uma aplicação MES (Manufacturing Execution System). As Figuras 3 e 4 mostram os equipamentos e aplicações do laboratório de manufatura avançada 4.0.

Figura 3 - Equipamentos contidos no laboratório de indústria 4.0.

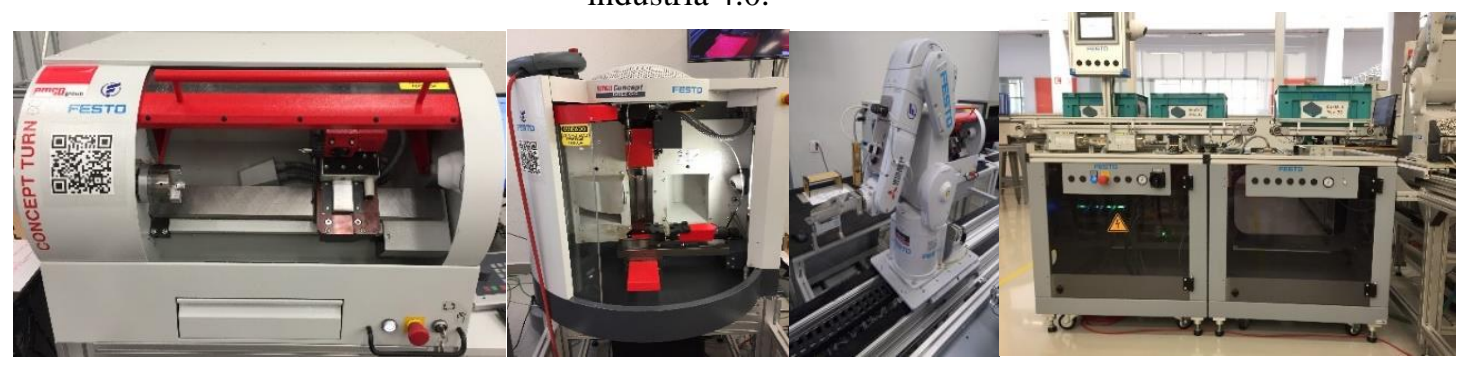

Fonte: Autoria própria.

Figura 4 - Funcionamento do sistema MES com o módulo 4.0 .

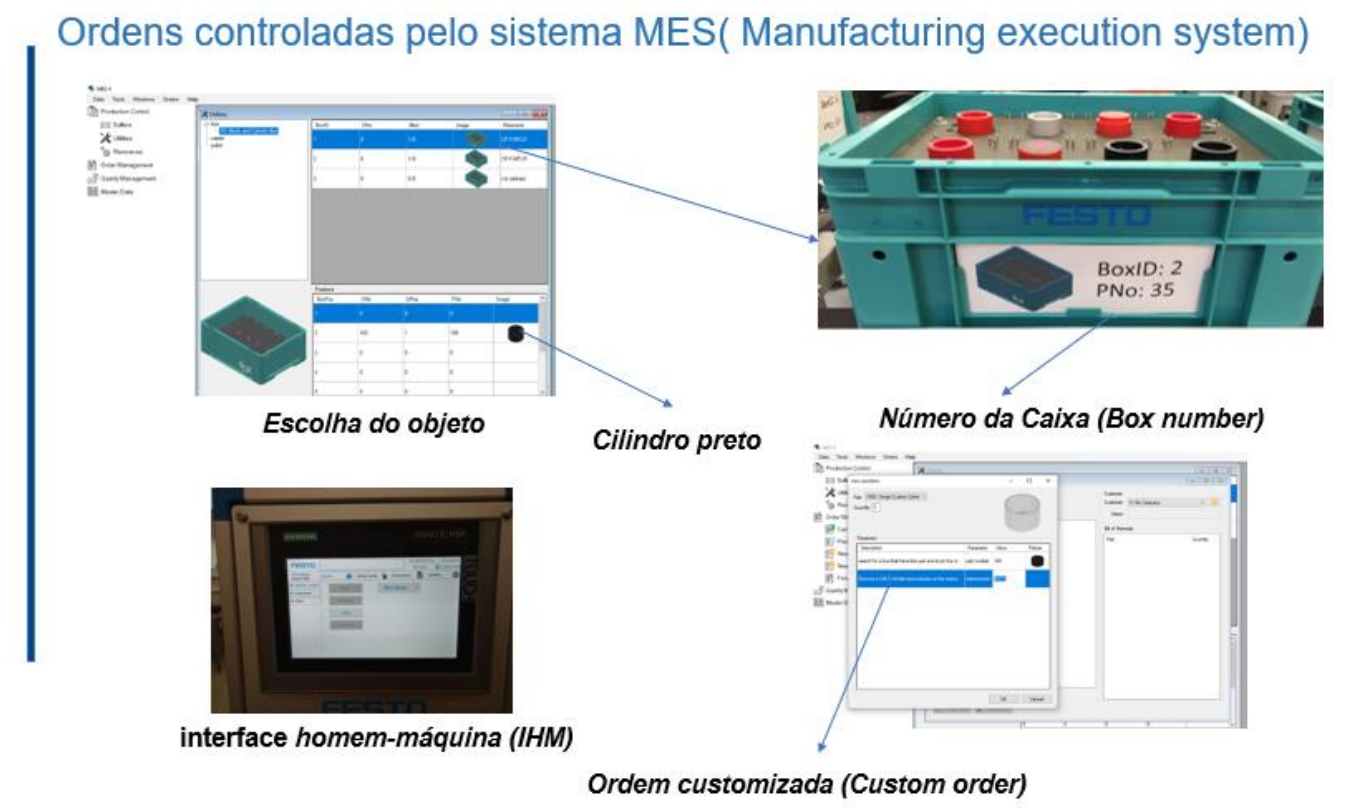

Fonte: Autoria própria.

Esses novos recursos possibilitaram a reformulação das grades dos cursos de graduação e pós-graduação, incorporando novos projetos, experimentos e oportunidades dentro da instituição.

Um dos projetos e experimentos incorporados ao planejamento foi o desenho por programação Melpha Basic V (linguagem de programação). Uma garra para suporte a canetas piloto foi desenvolvida no FABLAB (laboratório de prototipagem) por meio de uma impressora 3D. A construção da garra possibilitou elaborar uma metodologia utilizando o braço robótico presente no laboratório e o software CIROS®, disponibilizado pela fabricante Mitsubishi, é usado para a simulação do desenho e compilação do algoritmo. A Figura 5 mostra o experimento em sua realização. 


\section{COBENGE COOBENGE 2020}

Figura 5 - Experimento de um desenho utilizando a linguagem Melpha Basic V.

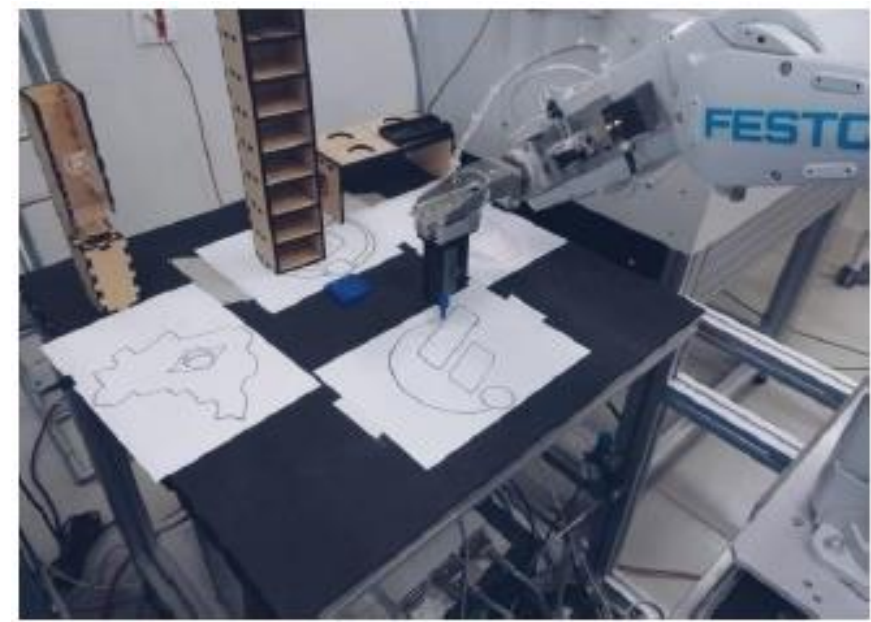

Fonte: Autoria própria.

O experimento acima foi aderido à grade curricular de engenharia mecatrônica, para entendimento de coordenadas e linguagem de programação voltada ao braço robótico em questão.

\section{ANÁLISE DE PROGRESSO E ATIVIDADES DESENVOLVIDAS}

A maior preocupação da instituição desde o início das atividades no laboratório era como explorar os recursos junto aos cursos que oferecia, as áreas que mais usufruíram do laboratório foram de engenharia mecânica e mecatrônica na parte de graduação. Na outra vertente, na parte de pós-graduação, os cursos que mais exploraram o laboratório foram de manufatura $4.0 \mathrm{e}$ controle e automação. A Figura 6 mostra a quantidade de estudantes que estiveram no laboratório.

Figura 6 - Estudantes que utilizaram o

laboratório.

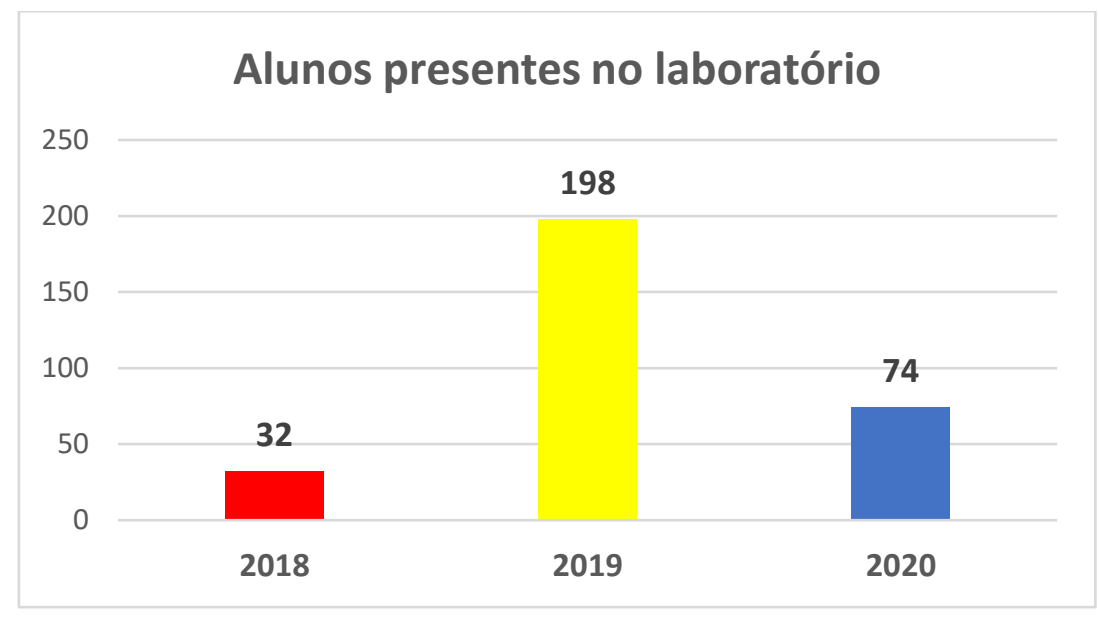

Fonte: Autoria própria. 
Mesmo que, quando comparado ao número total de estudantes é um número pequeno, deve-se considerar que os cursos estão passando por mudanças de grades para adaptação à essas novas exigências de mercado, então esse número deve aumentar ao longo do tempo. A organização também aumentou sua capacidade em cursos de pós-graduação, oferecendo cursos voltados a 4.0. A Figura 7 mostra a utilização do laboratório pelos cursos de Controle e Automação, assim como o de Manufatura 4.0.

Figura 7 - Utilização do laboratório pelos cursos de pós-graduação.

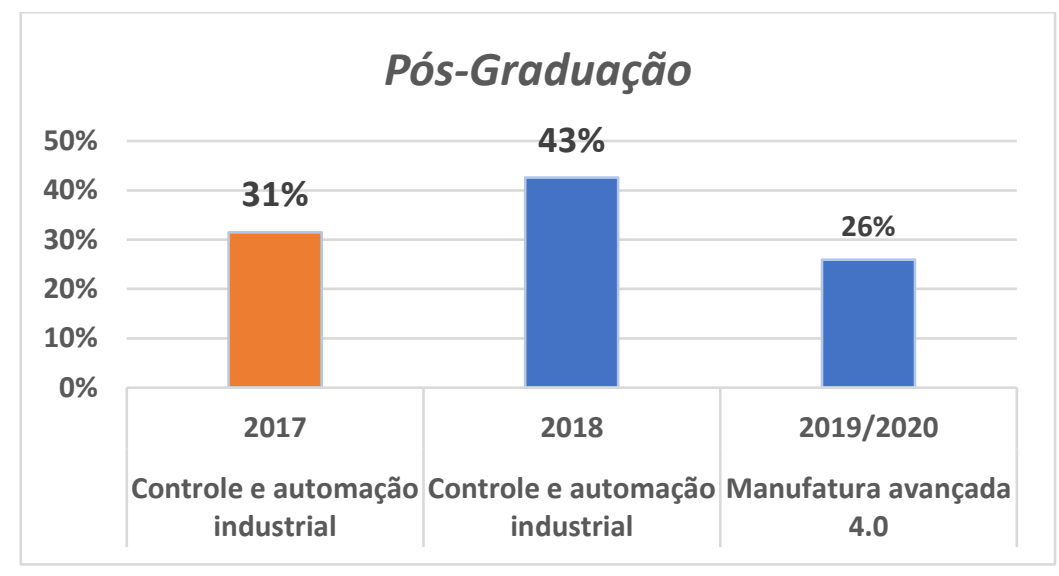

Fonte: Autoria própria.

Além de promover recursos tecnológicos, o laboratório também serve de vertente para elaboração de projetos. Foram elaborados um total de 31 projetos, no qual 15 projetos estão em desenvolvimento e 16 foram finalizados. A Figura 8 mostra os status dos projetos.

Figura 8 - Projetos finalizados e em andamento.

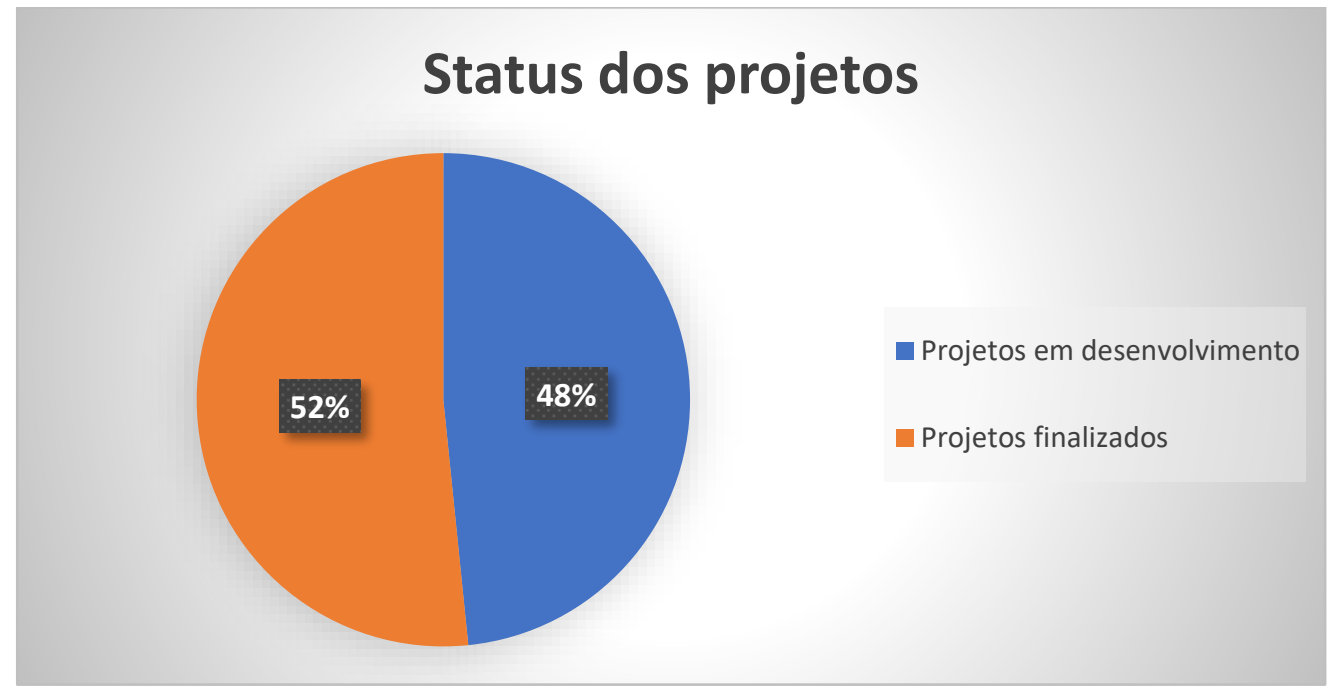

Fonte: Autoria própria.

É importante mencionar que alguns projetos dependem de recursos financeiros para executá-los. Esses se encontram em desenvolvimento em busca de parcerias e os trabalhos de conclusão de curso (TCCs) não estão inclusos nestes números. 
Como próximos passos a instituição visa aumentar o número de parcerias e projetos, a ideia é que a equipe possa fazer algo que agregue mais valor e traga retorno tecnológico para a instituição e parceiros. Um dos novos projetos que foi iniciado recentemente é de um KIT 4.0 que é idealizado para atender necessidades das indústrias, proporcionando dados importantes como o de temperatura, pressão, vazão, vibração, etc. Outro projeto em destaque é a possibilidade de integração de uma Máquina de Medir por Coordenadas (MMC) junto à célula de manufatura, ramificando as possibilidades com o aumento de rastreabilidade utilizando a tecnologia RFID.

A instituição também visou implementar workshops e palestras voltados ao tema de indústria 4.0. No total foram seis palestras e workshops proporcionados pelos participantes do núcleo e empresas parceiras.

\section{CONSIDERAÇÕES FINAIS}

A pesquisa visou demonstrar os benefícios de se ter um laboratório voltado a indústria 4.0 no ensino superior, mostrou como este se encaixa no cenário atual ajudando a instituição criar valores próprios.

Enfatizou que a aplicação de aprendizagem no contexto da indústria 4.0 é de suma importância o trabalho prático "hands-on", visto que para se formar profissionais voltados à esta nova demanda do mercado, o conhecimento teórico precisa ser aplicado de alguma forma, onde o laboratório de manufatura 4.0 tem um papel relevante nesse contexto.

O Centro Universitário Facens se preocupa com a capacitação e formação dos seus estudantes, mesmo sabendo dos desafios de se formar um profissional nos dias de hoje, a instituição tem investido cada vez mais em novas e melhores tecnologias com o objetivo de manter seu corpo estudantil atualizado e condizente com as atuais e futuras demandas de mercado.

\section{Agradecimentos}

Agradecimento especial ao Centro Universitário Facens por todo o apoio concedido aos professores e alunos no desenvolvimento de pesquisas e na formação continuada.

\section{REFERÊNCIAS}

ABELE, Eberhard et al. Learning factories for research, education, and training. Hochschule Reutlingen, 2015.

COSTA, Cesar da. Indústria 4.0: o futuro da indústria nacional. Pós-Graduação em Revista, São Paulo, v. 1, n. 4, p. 5-14, 2017.

COSTACHE, A. G.; POPA, C. L.; DOBRESCU, T.; COTET, C. E. The gap between the knowledge of virtual enterprise actor and knowledge demand of industry 4.0. In: 28th DAAAM international symposium on intelligent manufacturing and automation, Vienna, p.743$749,2017$.

KOLB, D. A. Experiential Learning. Englewood Cliffs, NJ: PrenticeHall. 1984. 
LEAL, Lorenna F.; FLEURY, André; ZANCUL, Eduardo. Starting up a Learning Factory focused on Industry 4.0. Procedia Manufacturing, v. 45, p. 436-441, 2020.

MÜLLER, Julian M.; BULIGA, Oana; VOIGT, Kai-Ingo. The role of absorptive capacity and innovation strategy in the design of industry 4.0 business Models-A comparison between SMEs and large enterprises. European Management Journal, 2020.

PUNDAK, David; ROZNER, Shmaryahu. Empowering engineering college staff to adopt active learning methods. Journal of Science Education and Technology, v. 17, n. 2, p. 152163, 2008.

SCHUSTER, Katharina et al. Preparing for industry 4.0-collaborative virtual learning environments in engineering education. In: Engineering Education 4.0. Springer, Cham, 2016. p. 477-487.

SEGATTO-MENDES, Andrea Paula. Análise do processo de cooperação tecnológica universidade-empresa: um estudo exploratório. 1996. Tese de Doutorado. Universidade de São Paulo.

TARALLI, Carmine. Universidade-Indústria: parceria na inovação. Revista USP, n. 25, p. 4247, 1995.

\title{
BENEFITS OF A LABORATORY OF INDUSTRY 4.0 IN HIGHER EDUCATION
}

\begin{abstract}
The concept about industry 4.0 evolved various other concepts, however for theses be developed in a new ecosystem, it needs to have a suitable structure. The content about this research brings to show the structural scenarios that contain in a Brazilian university centre, considering how these technological resources can help the organization reach the market reference. The Engineering University Sorocaba (Facens) contains an industry 4.0 laboratory, which will be used as a base study aiming to know how the universities transmit knowledge. The graduation and undergraduate courses were analyzed in respect to advanced technologies, 270 students enjoyed the infrastructure in 2018 and 2019. The undergraduate courses that has used the infrastructure was mechanical engineering, production engineering and mechatronic engineering. In regard to graduation courses the advanced manufacturing 4.0 and automation and control has taken part using the laboratory. The students impacted from this environment has increased 55\% when it is compared in relation to 2018 and 2019. The active learning methodology is one of the factors that most contributed to improving the learning method inside the laboratory it was designed totally towards teaching, from table settings to lesson planning. The space also contributed to the development academic projects, with a total of 31 proposed projects, of which 16 were completed and 15 are under development.
\end{abstract}

Keywords: industry 4.0. research. market. 